\title{
Using Machine Learning to Establish the Relationship between Die Casting Parameters and the Casting Quality
}

\author{
Pr. S. Henry Juang1, Yi-Ning Huang², David A. Kafando ${ }^{3}$ \\ ${ }^{1,2}$ National Taiwan Ocean University, Department of Mechanical and Mechatronic Engineering \\ No 2 Peining Rd., Zhongzheng Dist., Keelung City, Taiwan (ROC) \\ ${ }^{1}$ shjuang@mail.ntou.edu.tw; ${ }^{2}$ love9721227@gmail.com \\ 3davidanselmek@gmail.com
}

\begin{abstract}
The die casting process is highly automated and computerized. Nowadays, the machines are able to display dozens of data each cycle of the process with few setting parameters. The daily data remain unexploited in the industry. In this experiment, there are six setting parameters for the machine, such as cylinder pressure, high speed, high-speed switching point, intensification pressure starting position, injection delay, and biscuit thickness. The purpose of this research is to use machine learning to build two models, model 1 establishes the relationship between die casting machine setting parameters and the machine response (displayed) parameters via polynomial regression and the $\mathrm{R}$ square is used to evaluate the model, and model 2 is built using support vector machine algorithm, predicts the quality of die castings based on machine response parameters. The two models are then combined, and it allows the foundry men to adjust the machine parameters to improve the quality of die-casting parts. The experimental results of model 1 show that R squared greater or equal to 0.5 means that the setting parameters and the reaction parameters have a certain correlation. After cross-validation of model 2 , the model is stable and the accuracy rate can reach $74 \%$, with a small amount of data under the circumstances, it has reached the applicable standard. This research results are based on two data sets provided by diecasters A and B to establish and verify the models.
\end{abstract}

Keywords: machine learning, polynomial regression, support vector classification, casting quality, parameter adjustment.

\section{Introduction}

In response to Industry 4.0, Taiwan government promotes " $5+2$ " innovative industry, among which smart machinery, it aims to promote the introduction of smart technology in the machinery industry, establish a smart machinery ecosystem, provide innovative products and services, and promote the transformation and upgrading of Taiwan's industry [1].

The foundry industry is the basis of the machinery industry. The high-pressure casting is a highly automated method suitable for mass production in the foundry industry, which is quite suitable for the development of intelligent die-casting technology. The intelligent die-casting technology system is sequenced into perception, thinking and action, as shown in Figure 1. Among them, the sensing layer using sensors to measure the casting pressure in the mold cavity, the surface temperature of the mold cavity, and the temperature of molten alloy, etc., are collected to the database through the Internet of Things (IoT). In the thinking layer, the data collected by the sensing layer mark the data and divide the data into training group and testing group, using machine learning to build models. The action layer applies the prediction model established by the thinking layer to die casting production, and adjust the parameters according to the model results to achieve the goal, high efficiency, stable quality and low energy consumption requirements $[2,3]$. 


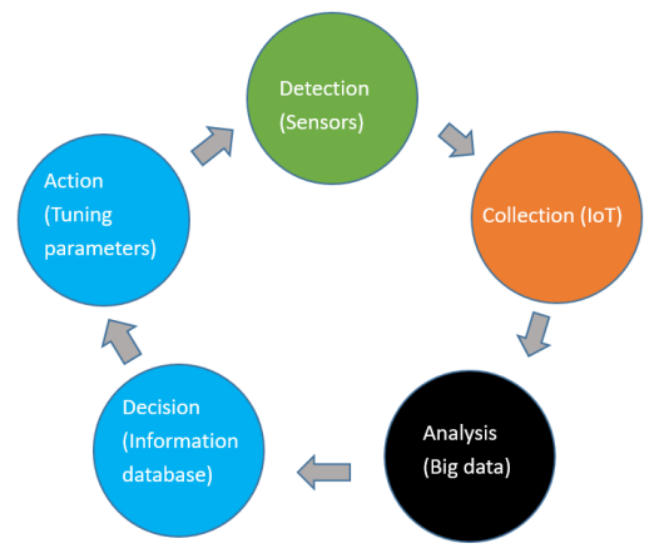

Fig. 1: Smart Die Casting Technology System

The die casting industry never stop innovating and integrating new technologies into the process and production lines. Yarlagadda and Chiang [4] established an artificial neural networks (ANN) based model that allow to obtain the process parameters for the high pressure die casting process using three different training algorithms, such as the error backpropagation algorithm, the momentum and adaptive learning algorithm. Their investigation showed that the casting conditions, e.g., melt temperature, die temperature, injection pressure and injection time, dominate the quality of the casting. Anijdan et al. [5] in order to optimize the process parameters associated with the minimum porosity rate in Al-Si casting alloys, developed a combined genetic algorithm-artificial neural network (GA-ANN) method. Tsoukalas [6] developed linear regression and genetic algorithm (GA) as an effective method to find the optimal process parameters associated with the minimum percentage of porosity of $\mathrm{AlSi}_{9} \mathrm{Cu}_{3}$ aluminum alloy castings. Part of the die-casting industry relies on the diecasting master's experience and traditional trial-and-error methods to adjust process parameters to improve the quality of castings. Because there is no systematic basis for adjusting the parameters, it consumes a lot of production costs and time. In addition, new die-casting personnel take time to obtain knowledge and experience. The purpose of this research is to use machine learning to establish a prediction model of the casting quality based on machine process parameters.

\section{Methodology}

This research aims to use the programming language — Python 3 to construct a set of die-casting AI models with machine learning to predict the impact of the variation of die-casting process parameter on the quality of die-casting parts, and to monitor die-casting production information to improve die-casting yield. The actual research methodology chart shown in Figure 2.

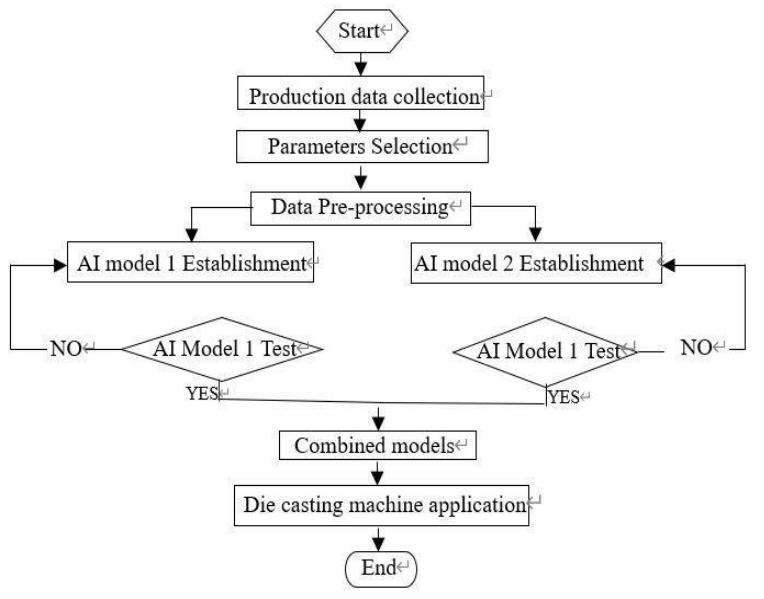

Fig. 2: Research flow chart. 


\subsection{Select Feature Parameters}

The die-casting industry has always pursued new technologies to meet customers' high-quality requirements for the products. In addition, process parameters are also the main factors affecting the quality of die-casting parts. There are about dozens of displayed parameters of the die-casting machine, of which only some parameter directly affect the quality of diecasting parts. Therefore, the correlation analysis and literature reviews were used to select characteristic parameters to perform machine learning with more influential parameters.

\subsubsection{Correlation Analysis}

The correlation analysis is a method to study the direction and closeness of the interdependence between two or more random variables. The Pearson correlation coefficient mainly used to express the linear correlation strength between continuous variables [7]. The Pearson correlation coefficient was calculated using the Eq. (1). Table 1 shows the correlation between the density of castings and process parameters. After the correlation coefficient is taken as an absolute value, the greater the coefficient, the stronger the relationship with the density of the casting; A positive correlation coefficient indicating that the large the process parameter, the higher the casting density; on the contrary, the smaller the process parameter, the higher the casting density.

$$
r==\frac{\sum\left(x_{i}-\bar{x}\right)\left(y_{i}-\bar{y}\right)}{\sqrt{\sum\left(x_{i}-\bar{x}\right)\left(y_{i}-\bar{y}\right)}}
$$

where $r$ is the correlation coefficient; $x_{\mathrm{i}}$ and $\mathrm{y}_{\mathrm{i}}$ are the values of the $x$-variable and $\mathrm{y}$-variable in a sample, respectively; $\bar{x}$ and $\bar{y}$ denote the mean of the values of the $x$-variable and $y$-variable, respectively.

Table 1: Correlation between casting density and process parameters.

\begin{tabular}{|c|c|}
\hline & Density \\
\hline High speed & 0.030 \\
\hline Hydraulic pressure & 0.452 \\
\hline Casting pressure & 0.452 \\
\hline Biscuit thickness & 0.494 \\
\hline Clamping force & 0.188 \\
\hline Low speed time & 0.173 \\
\hline High speed time & -0.183 \\
\hline Intensification pressure time & 0.029 \\
\hline Pouring time & -0.404 \\
\hline Low speed stroke & 0.198 \\
\hline High speed stroke & -0.366 \\
\hline Cycle stroke & -0.494 \\
\hline
\end{tabular}

\subsubsection{Literature Selection}

According to Pascal's principle, the casting pressure can be obtained from the cylinder pressure, cross-sectional area of injection cylinder and plunger tip, as shown in Eq. (2). When the casting pressure is increased, the quality of the casting can be improved; however, if the casting pressure is too high, it may cause problems such as poor casting size: too low may cause the casting to appear porous and other casting defects [8].

$$
\text { Casting pressure }=\frac{\text { Cylinder pressure } * \text { cylinder piston area }}{\text { Plunger tip area }}
$$

During the die-casting process, the plunger speed can be divided into two stages, low and high speed. The melt is pushed at a low speed to fill the shot sleeve, and then forced at a high speed to inject it into the mold cavity. Controlling the lowspeed speed can reduce the air entrapment in the sleeve. The gate speed affects the surface of the casting quality and also the mold life, the ideal gate speed can be obtained by controlling the high-speed. The melt reaches the gate at a position switching from low to high-speed. According to different products, the high-speed switching position can be adjusted to obtain a better casting quality. 
The intensification stage in the die casting process is the key affecting the mechanical properties of the casting. When the intensification starting time is too early, it affects the high speed and cause burrs in the die casting; if it is too late, the intensification effect may not be achieved. The biscuit thickness of the casting affects the pressure transmission in the intensification stage. Usually the biscuit thickness is maintained at $10 \sim 30 \mathrm{~mm}$. When the porosity rate requirements are strict, it would thicken about $2 \sim 5 \mathrm{~mm}$.

Based on the results of the correlation analysis, the process parameters that affect the quality of the castings are selected. The parameters with a correlation coefficient of 0.3 or more are selected as the characteristic parameters, such as the cylinder pressure, casting pressure. According to the above-mentioned Eq. (2), the casting pressure is calculated from the cylinder pressure, so the final characteristic parameters are the cylinder pressure, the biscuit thickness, the pouring time, the highspeed stroke and the overall stroke.

\subsection{Data Pre-Processing}

The key factors of the learning results of machine learning algorithms are the quality of the data and the amount of useful information contained. In order to improve the prediction results of the model, the data needs to be pre-processed. Common pre-processing method includes missing value processing by removing or replanting blanks or placeholders in the data table, category classification processing by encoding category labels as integer values, data feature scaling-mainly divided into two methods, regularization and standardization [9]. Normalization is to scale the feature data to [0,1] or [1, -1] without changing the original distribution.

In the present study the quality of castings is judged by density, and it is divided into categories such as good, acceptable, pending and defective rates according to the diecaster's needs. There are four categories, as shown in Table 2.

Table 2: Classification of casting quality.

\begin{tabular}{|c|c|}
\hline Category & Density $\left(\mathrm{g} / \mathrm{cm}^{3}\right)$ \\
\hline 1-Defective & $\rho<2.6810$ \\
\hline 2- Pending & $2.6810<\rho<2.6868$ \\
\hline 3-Acceptable & $2.6868<\rho<2.6977$ \\
\hline 4-Good & $\rho>2.6977$ \\
\hline
\end{tabular}

\subsection{Al Model Establishment}

The first model uses polynomial regression analysis to analyse the machine settings and machine response parameters to find the correlation between the two, the correlation is determined by the regression coefficient. The machine setting parameters in this study are only six parameters such as cylinder pressure, high speed, high-speed switching point, intensification pressure starting position, injection delay, and biscuit thickness. The second model uses the Support Vector Machine/ Support Vector Classification (SVM/SVC), to study the relationship between the machine displayed parameters and the porosity (quality) is established [10]. However, due to insufficient data provided by the diecaster, the establishment of model 1 is not yet completed. Only model 2 can be used to predict the casting quality with machine response parameters, and then model 1 can be used to understand the setting parameters that affect the response parameters for adjustment.

The polynomial regression analysis is used for modelling, which gives a better fit, as shown in Eq. (3) [11].

$$
y_{i}=\beta_{0}+\beta_{1} x_{i}+\beta_{2} x_{i}^{2}+\cdots+\beta_{p} x_{i}+\beta_{q} x_{i}^{2}+\varepsilon_{i}(i=1,2, \cdots, n)
$$

where $y_{i}$ represent the machine displayed parameters, $\beta_{0}$ to $\beta_{\mathrm{q}}$ are the regression coefficients and the independent variable $x_{i}$ represent the setting parameters.

Support vector machine is a supervised learning model for analyzing data in classification and regression and related learning algorithms, such as Support Vector Classification and Support Vector Regression. The goal is to find an optimal hyperplane to separate the data of different categories, as shown in Figure 3 [12]. 


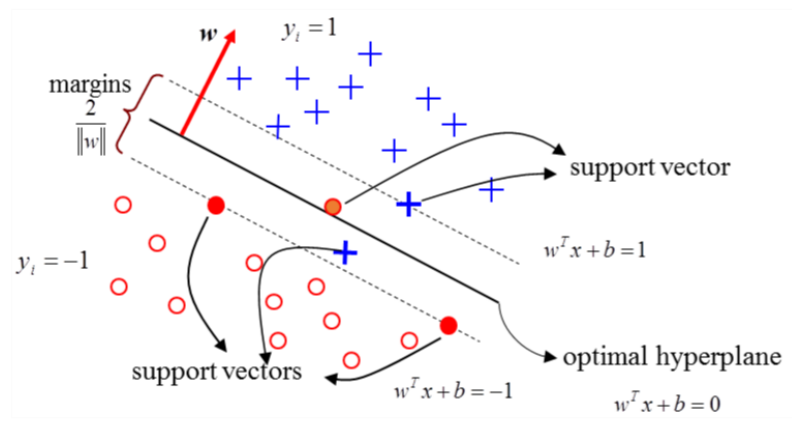

Fig. 3: Support Vector Machine [12].

\section{Results and Discussion}

In this study, machine learning was used to establish a casting quality prediction model, and two models were established separately and then integrated.

\subsection{Training Results of Model 1}

Model 1 is evaluated using the R-squared value. The larger the number, the better the fit of the regression model. The cylinder pressure, high-speed speed, high-speed switching point, pressurization start position, injection delay, and biscuit thickness are the inputs parameters; $y_{i}$ are the machine response parameters such as cylinder pressure, biscuit thickness, pouring time, high-speed stroke, and overall stroke. The determination coefficients of the final machine setting parameters and reaction parameters are shown in Table 3, and the polynomial regression equation is shown in Table 4.

Table 3: The fit of displayed parameters used in model 1 after normalization processing.

\begin{tabular}{|c|c|}
\hline Characteristic Parameters & R-square \\
\hline Cylinder pressure & 0.999 \\
\hline Biscuit thickness & 0.905 \\
\hline Pouring time & 0.57 \\
\hline High speed stroke & 0.686 \\
\hline Overall stroke & 0.752 \\
\hline
\end{tabular}

Table 4: The characteristic regression coefficient after normalization in polynomial regression analysis of model 1.

\begin{tabular}{|c|c|c|c|c|c|}
\hline & $y_{1}$ & $y_{2}$ & $y_{3}$ & $y_{4}$ & $y_{5}$ \\
\hline$x_{1}$ & 0.795 & 0.498 & -0.088 & 0.994 & 0.559 \\
\hline$x_{1}^{2}$ & 0.207 & -0.641 & 0.133 & -0.747 & -0.255 \\
\hline$x_{2}$ & 0.010 & 0.017 & 0.0047 & 0.141 & 0.131 \\
\hline$x_{2}^{2}$ & 0.010 & 0.017 & 0.0047 & 0.141 & 0.131 \\
\hline$x_{3}$ & -0.143 & -0.329 & 0.091 & -0.731 & -0.159 \\
\hline$x_{3}^{2}$ & 0.148 & 0.433 & -0.059 & 0.359 & 0.347 \\
\hline$x_{4}$ & 0.010 & 0.059 & 0.014 & 0.124 & 0.087 \\
\hline$x_{4}^{2}$ & 0.010 & 0.059 & 0.014 & 0.124 & 0.087 \\
\hline$x_{5}$ & -0.0013 & 0.044 & 0.071 & 0.129 & 0.101 \\
\hline$x_{5}^{2}$ & -0.0013 & 0.044 & 0.079 & 0.129 & 0.101 \\
\hline$x_{6}$ & 0.157 & 0.881 & -0.058 & 0.562 & -0.031 \\
\hline$x_{6}^{2}$ & -0.185 & -0.107 & 0.091 & -0.714 & -0.564 \\
\hline
\end{tabular}

Table 3 shows the R square value of the machine setting parameters and displayed parameters after normalization. They are all greater than 0.5 , indicating that these displayed parameters have a certain relationship with the setting parameters. Table 4 shows the equation of polynomial regression analysis. According to Table 4, it can be seen that the machine setting parameters affect the reaction parameters. The larger the coefficient, the greater the influence. Among them, the cylinder 
pressure of the setting parameter has a greater influence on the cylinder pressure, the thickness of the biscuit thickness, the high-speed stroke, and the overall stroke of the response parameters.

\subsection{Training Results of Model 2}

This study uses SVC algorithm to establish model 2, taking machine response parameters as input and casting quality as output. The data provided by diecaster A has two die casting data, which are mixed for training. However, because the trained model is too simple or too complex, there are problems with low bias or high variance. In order to obtain a more reliable model, this study uses k-fold cross-validation [13]. The final cross-validation model accuracy rate was $74 \%$.

\subsection{Influence of Parameter Adjustment on Casting Quality}

Combining model 1 and model 2 can not only predict the product quality of the die-casting process, but also find the main settable parameters that affect the quality of the finished product, and provide the setting parameter adjustment direction to reduce the traditional trial and error method that consumes a lot of production costs and time, and improve product yield. According to the results of model 1, it allows to select the parameters that have R square equal or greater to 0.9 , and observe that it is applied to model 2. It can be seen that increasing the cylinder pressure and the biscuit thickness of the response parameters can increase the yield. According to the model 1, adjusting the cylinder pressure affects the cylinder pressure of the displayed parameter; changing the biscuit thickness can adjust the biscuit thickness, the pressure of the cylinder and the high-speed switching point in sequence. Taking the prediction result of cylinder pressure as an example, as shown in Table 5 , some of the classification results are bad, and it is speculated that the error is caused by imperfect data. According to Model 1, if the cylinder pressure of the reaction parameter is to be modified, the cylinder pressure, the biscuit thickness and the high-speed switching point of the set parameters can be adjusted in order to improve the quality of the casting.

Table 5: Predictive classification results of adjusting cylinder pressure

\begin{tabular}{|c|c|c|c|c|c|}
\hline $\begin{array}{c}\text { Hydraulic } \\
\text { pressure }(\mathrm{MPa})\end{array}$ & $\begin{array}{c}\text { Biscuit } \\
\text { thickness (mm) }\end{array}$ & $\begin{array}{c}\text { Pouring } \\
\text { time(s) }\end{array}$ & $\begin{array}{c}\text { High speed } \\
\text { stroke(mm) }\end{array}$ & $\begin{array}{c}\text { Overall cycle } \\
\text { stroke (mm) }\end{array}$ & $\begin{array}{c}\text { Quality } \\
\text { classification }\end{array}$ \\
\hline 180 & 18 & 11 & 94 & 345 & 1 \\
\hline 183 & 18 & 11 & 94 & 345 & 1 \\
\hline 190 & 18 & 11 & 94 & 345 & 3 \\
\hline 200 & 18 & 11 & 94 & 345 & 3 \\
\hline 210 & 18 & 11 & 94 & 345 & 1 \\
\hline 220 & 18 & 11 & 94 & 345 & 3 \\
\hline 230 & 18 & 11 & 94 & 345 & 4 \\
\hline 240 & 18 & 11 & 94 & 345 & 3 \\
\hline 250 & 18 & 11 & 94 & 345 & 1 \\
\hline 260 & 18 & 11 & 94 & 345 & 3 \\
\hline 270 & 18 & 11 & 94 & 345 & \\
\hline
\end{tabular}

\subsection{Application to Different Data Sets}

There are more than 7000 die-casting data sets provided by diecaster B. The die-casting data of this diecaster only provides machine displayed parameters and casting quality classification results. Therefore, following the same method of establishing model 2 in this study, the same prediction model is established. The correlation analysis is used to select the characteristic parameters, and the correlation between the displayed parameters and the product quality results is shown in Table 6. The selection of the characteristic parameters takes into account the Pearson correlation coefficient greater than 0.3 (>0.3). It is known that the casting pressure is calculated from the cylinder pressure, so the final characteristic parameters are the cylinder pressure, low-speed speed, high-speed speed, and hydraulic oil temperature as input. The casting quality result is divided into two types good and bad. Taking $80 \%$ of the data set provided by diecaster B as the training group and $20 \%$ as the test group, the accuracy rates are $99.58 \%$ and $99.8 \%$, respectively. 
Table 6: Correlation between selected parameters and good product results.

\begin{tabular}{|c|c|}
\hline & Good product \\
\hline Casting pressure & -0.898 \\
\hline High speed & -0.684 \\
\hline Low speed & -0.717 \\
\hline Intensification pressure starting time & -0.050 \\
\hline Cycle time & 0.094 \\
\hline Biscuit thickness & 0.178 \\
\hline Low speed stroke & 0.003 \\
\hline Oil temperature & -0.504 \\
\hline $2^{\text {nd }}$ phase speed & -0.167 \\
\hline $3^{\text {rd }}$ phase speed & -0.174 \\
\hline $1^{\text {st }}$ phase speed & -0.033 \\
\hline Spray 1 time & $2.59 \mathrm{E}-16$ \\
\hline Spray 2 time & $2.59 \mathrm{E}-16$ \\
\hline Blow 2 time & $2.59 \mathrm{E}-16$ \\
\hline Cylinder pressure & -0.898 \\
\hline
\end{tabular}

\section{Conclusion}

The following conclusions were obtained from the study results:

1. According to the results of the polynomial regression analysis of the model, the machine setting parameters and displayed parameters such as cylinder pressure, biscuit thickness, feeding time, high-speed stroke, and overall stroke have a high R squared value, respectively $0.999,0.905,0.57,0.686$ and 0.752 . All are above 0.5 , indicating that those machine setting parameters and the display parameters have a certain correlation.

2. After the cross-validation of model 2 , the model has reached stability and the accuracy rate is $74 \%$. Using the data set of diecaster B which has a large amount of data and the quality of castings is only classified into two categories, the same model reaches $99 \%$ accuracy.

3. This study hopes to combine model 1 and model 2, and finally set the parameters of the machine as input and casting quality as output to establish a complete model for predicting die casting quality. However, due to insufficient data, only model 2 can be used to find out the impact the response parameters of casting quality are adjusted according to the influence of the model 1 machine setting parameters on the response parameters. In addition, because the amount of data is too small, this study is only a preliminary machine learning model, and it is still unable to achieve self-learning.

4. According to the application of the process parameters in model 2, increasing the cylinder pressure and the thickness of biscuit of the response parameters can increase the yield. To change the cylinder pressure, it must first adjust the cylinder pressure of the set parameter; changing the thickness of biscuit can adjust in order the thickness of biscuit, cylinder pressure and high-speed switching point. Reducing the overall stroke of the response parameters can increase the yield. Adjust the overall stroke to sequentially adjust the cylinder pressure, high-speed speed, high-speed switching point, injection delay, and tip thickness of the set parameters.

\section{Acknowledgements}

This study is the project sponsored by the Ministry of Science and Technology (MOST) in Taiwan, with project numbered MOST-180-2221-E-019-MY3. Without the support of MOST, it would not fulfil to carry out this project smoothly. It is also grateful to the diecasters $\mathrm{A}$ and $\mathrm{B}$ for the die casting machine data and casting quality. Also thanks to Pr. Dong-Lin Li, Department of Electrical Engineering at NTOU, for his guidance and advice on machine learning.

\section{References}

[1] https://www.ey.gov.tw/Page/5A8A0CB5B41DA11E/18ef26a4-5d05-4fb3-963e-6b228e713576 2019.

[2] H. Lee, K. Ryu, and Y. Cho, "A framework of a smart injection molding system based on real-time data", Procedia Manufacturing 11:1004-1011, 2017, DOI:10.1016/j.promfg.2017.07.206

[3] Y. Zhao, F. Qian, Y. Gao, "Data driven die casting smart factory solution", ICSEE/IMIOT, CCIS, vol. 923, pp13-21. Springer, Singapore, 2018. 
[4] K. Prasad and D.V. Yarlagadda, "Prediction of die casting process parameters by using an artificial neural network model for zinc alloys", International Journal of Production Research, 38, 119-139, 2010.

[5] S. H. M. Anijdan, A. Bahrami, H. R. M. Hosseini, and A. Shafyei, "Using genetic algorithm and artificial neural network analyses to design an Al-Si casting alloy of minimum porosity", Mater. Des. 27(7), 605-609, 2006.

[6] V. D. Tsoukalas, "Optimization of porosity formation in $\mathrm{AlSi}_{9} \mathrm{Cu}_{3}$ pressure die castings using genetic algorithm analysis”, Mater. Des., 29(10), 2027-2033, 2008.

[7] M. Franzese and A. Iuliano, "Correlation analysis, encyclopedia of bioinformatics and computational biology", Vol.1, pp.706-721. 2019.

[8] S. H. Juang, Fabrication Technology of Intelligent Die Casting Molds ( $2^{\text {nd }}$ ed.), Wunanbooks, Taiwan, 2013.

[9] W. McKinney, Translated by Zhang Jingwen, Python data analysis ( $2^{\text {nd }}$ ed.), GOTOP Information Inc., Taiwan, 2018.

[10] M. Fan and C. Li, "Python machine learning and practice", Tsinghua University Press, China, 2016.

[11] Python programming and data analysis, Princeton International Publishing Co., Ltd., Taiwan, 2019.

[12] T. Huang, "Machine Learning-support vector machine (SVM) detailed derivation", 2018, [online]. https://chih-shenghuang821.medium.com/\%E6\%A9\%9F\%E5\%99\%A8\%E5\%AD\%B8\%E7\%BF\%92$\% \mathrm{E} 6 \% 94 \% \mathrm{AF} \% \mathrm{E} 6 \% 92 \% 90 \% \mathrm{E} 5 \% 90 \% 91 \% \mathrm{E} 9 \% 87 \% 8 \mathrm{~F} \% \mathrm{E} 6 \% \mathrm{~A} 9 \% 9 \mathrm{~F}-$ support-vector-machine-svm$\% \mathrm{E} \%$ A9\%B3\%E7\%B4\%B0\%E6\%8E\%A8\%E5\%B0\%8E-c320098a3d2e

[13] S. Raschka and L. I. Liu, Compiled by J. H. Wu, Python machine learning, DrMaster Press Co., Ltd, Taiwan, 2016. 